\title{
CXCR4 Mediates Enhanced Cell Migration in CALM-AF10 Leukemia
}

\author{
Shelby A. Fertal ${ }^{1 \dagger}$, Sayyed K. Zaidi ${ }^{2,3+}$, Janet L. Stein ${ }^{2,3}$, Gary S. Stein ${ }^{2,3}$ \\ and Jessica L. Heath ${ }^{1,2,3^{*}}$
}

1 Department of Pediatrics, Larner College of Medicine, University of Vermont, Burlington, VT, United States, 2 Department of Biochemistry, Larner College of Medicine, University of Vermont, Burlington, VT, United States, ${ }^{3}$ University of Vermont Cancer Center, Burlington, VT, United States

OPEN ACCESS

Edited by:

Chung Hoow Kok

University of Adelaide, Australia

Reviewed by:

Federica Barbieri,

University of Genoa, Italy

Stefania Scala

IRCCS Istituto Nazionale Tumori

Napoli "G. Pascale", Italy

*Correspondence: Jessica L. Heath

jessica.l.heath@med.uvm.edu

${ }^{\dagger}$ Present address: Shelby A. Fertal,

Department of Cell and Regenerative Biology, University of Wisconsin,

Madison, WI, United States

Sayyed K. Zaidi,

Senior Scientific Writer, American Association for Cancer Research, Philadelphia, PA, United States

Specialty section:

This article was submitted to Hematologic Malignancies,

a section of the journal

Frontiers in Oncology

Received: 12 May 2021 Accepted: 07 December 2021 Published: 05 January 2022

Citation:

Fertal SA, Zaidi SK, Stein JL,

Stein GS and Heath JL (2022)

CXCR4 Mediates Enhanced Cell Migration in CALM-AF10 Leukemia.

Front. Oncol. 11:708915.

doi: 10.3389/fonc.2021.708915
Leukemia transformed by the CALM-AF10 chromosomal translocation is characterized by a high incidence of extramedullary disease, central nervous system (CNS) relapse, and a poor prognosis. Invasion of the extramedullary compartment and CNS requires leukemia cell migration out of the marrow and adherence to the cells of the local tissue. Cell adhesion and migration are increasingly recognized as contributors to leukemia development and therapeutic response. These processes are mediated by a variety of cytokines, chemokines, and their receptors, forming networks of both secreted and cell surface factors. The cytokines and cytokine receptors that play key roles in CALM-AF10 driven leukemia are unknown. We find high cell surface expression of the cytokine receptor CXCR4 on leukemia cells expressing the CALM-AF10 oncogenic protein, contributing to the migratory nature of this leukemia. Our discovery of altered cytokine receptor expression and function provides valuable insight into the propagation and persistence of CALM-AF10 driven leukemia.

Keywords: CXCR4, CXCL12, leukemia, migration, adhesion, CALM-AF10 leukemia

\section{INTRODUCTION}

The $t(10 ; 11)(p 13 ; q 14-21)$ chromosomal translocation results in the fusion of CALM (encoding the gene for the clathrin assembly lymphoid myeloid protein, also known as phosphatidylinositolCALM (PICALM)) to MLLT10 (also known as AF10) and is a recurrent genetic abnormality in acute lymphoid and myeloid leukemia. Nearly all of each individual gene is retained in this translocation. The endogenous CALM protein is primarily involved in intracellular trafficking. It associates with AP2 and clathrin, and functions to stabilize the clathrin-coated endocytic pit, allowing for efficient cellular uptake of growth factors and other receptor-bound molecules (1). Haploinsufficiency of CALM, as is found in cells expressing CALM-AF10, results in decreased endocytic efficiency (2). It has been reported that CALM contains a nuclear export signal (NES), allowing interaction with the CRM1/XPO1 nuclear export receptor. The NES is retained in the CALM-AF10 fusion construct, where it facilitates nucleocytoplasmic shuttling, and is necessary for leukemogenesis (3). AF10 is a transcription factor, binding to DNA through its leucine zipper domain, which is necessary for leukemogenesis (4-6). AF10 binds Dot1L, the only known H3K79 methyltransferase, and recruits Dot1L to its target genes, including the HOXA gene cluster (7). Upregulation of HOXA genes in CALM-AF10 leukemias is a driving factor in CALM-AF10 mediated leukemogenesis $(8,9)$. 
The CALM-AF10 translocation was first identified in a patient with histiocytic sarcoma but is more commonly seen in T acute lymphoblastic leukemia (T-ALL), where it makes up about $15 \%$ of pediatric and adult cases (10-12). CALM-AF10 translocated $\mathrm{T}$-ALL is characterized by the presence of extramedullary disease, CNS relapse, and poor response to therapy (12). This translocation is also seen in acute myeloid leukemia (AML), where it is associated with bulky hepatosplenomegaly, mediastinal disease, and central nervous system (CNS) leukemia (13).

Extramedullary and CNS leukemia development relies on migration of the leukemia cell out of the bone marrow compartment, and adhesion of the leukemia cells to cells of the target organ (CNS, liver, spleen, or lymph nodes). Multiple proteins have been implicated in this process, including several members of the integrin and chemokine families (14-16). More recently, interactions between leukemia cells and components of the bone marrow microenvironment have been shown to be critical for leukemic cell survival as well as resistance to chemotherapy [reviewed in (17)]. Interactions of leukemic cells with their microenvironment are mediated both by direct cellular contact and via soluble factors. One such factor is C-X-C motif chemokine receptor 4 (CXCR4), a cell surface G-protein coupled chemokine receptor that is bound and activated by $\mathrm{C}-\mathrm{X}-\mathrm{C}$ motif chemokine ligand 12 (CXCL12). CXCL12 can also enter the vascular system, and its interaction with CXCR4+ circulating hematopoietic stem cells (HSCs) is vital for HSC homing to the bone marrow compartment (18). The CXCR4/CXCL12 interaction plays a role in malignant hematopoiesis as well. Binding of CXCL12 to CXCR4 on leukemia cells activates multiple proliferative and survival pathways (reviewed in (19)), and high CXCR4 expression on leukemic blasts has been identified as a poor prognostic factor in both ALL and AML $(20,21)$. Finally, increased CXCR4 expression predicts the development of extramedullary disease in pediatric ALL (22).

We report an increase in cell surface expression of CXCR4, and a concomitant increase in the migratory behavior of cells transformed by CALM-AF10. Using both human and mouse cell line models, we examine the relationship between CXCR4 expression and phenotype in CALM-AF10 transformed leukemias. Surprisingly, we do not find synergistic effect of CXCR4 inhibition with traditional chemotherapy in these leukemias, strongly suggesting caution in the broad use of these inhibitors, and a more tailored approach to this targeted therapy.

\section{METHODS}

\subsection{Cell Culture}

Human leukemia cell lines were cultured in RPMI 1640 (Fisher Scientific, Waltham, MA, USA) supplemented with 100 units/ $\mathrm{mL}$ penicillin, and 100ug/mL streptomycin. U937 and Fujioka cells were additionally supplemented with $10 \%$ fetal bovine serum (Atlanta, USA) and 2mM L-glutamine. Kasumi-1 cells were additionally supplemented with $20 \%$ fetal bovine serum and
MEM non-essential amino acids. Human mesenchymal stem cells (hMSC) were cultured in $\alpha$-MEM (ThermoFisher Scientific, Waltham, MA, USA) media supplemented with $16.5 \%$ FBS, $2 \mathrm{mM}$ L-glutamine, 100 units/mL penicillin, and $100 \mathrm{ug} / \mathrm{mL}$ streptomycin. Murine leukemia cell lines were derived from cells isolated from the marrow of leukemic mice (a generous gift from Catherine Lavau, Duke University, North Carolina). Hoxa9-Meis1 and CALM-AF10 transformed cells were cultured in RPMI 1640 with $20 \%$ fetal bovine serum (Atlanta, USA), 100units $/ \mathrm{mL}$ of penicillin and streptomycin, and $10 \mathrm{ng} / \mathrm{mL}$ mouse recombinant IL-3 (Stem Cell Technologies). Murine embryonic fibroblasts (MEFs) were generated from Picalm ${ }^{\text {fit1-5R }}$ E14 embryos, immortalized with SV40, as previously described (2). Plat-E cells were transiently transfected with the MSCVIRES-GFP encoding CALM-AF10 (3), or the MSCV-IRES-GFP empty control vector using calcium phosphate. MEFs were then retrovirally infected with these constructs by co-culture with filtered Plat-E supernatant. Infection efficiency was measured by flow cytometry at the time of cell line genesis, and intermittently confirmed using fluorescent microscopy and western blot analysis. All cell lines were incubated at $37^{\circ} \mathrm{C}$ with 5 percent $\mathrm{CO}_{2}$. All human cell lines and were validated continually by assessment of morphology and annually by short tandem repeat (STR) analysis. Murine cell lines were validated continually by assessment of morphology in cell culture and periodically by western blot assessment of the presence or absence of the CALMAF10 fusion protein. In addition, all cells were confirmed negative for Mycoplasma infection with the Mycoalert detection kit (Lonza, Basel, CH) annually, or if any morphologic or growth changes were identified.

\subsection{Immunofluorescence}

Samples of $5 \times 10^{5}$ cells per condition (CXCR4 stained and negative control) for human and murine cell lines were spun down at $1500 \mathrm{rpm}$ for 5 minutes at room temperature, washed twice with PBS and fixed with 3.7\% formaldehyde while rotating for 10 minutes. Fixed cells were washed twice with PBS, and resulting pellets were resuspended in $200 \mathrm{uL}$ of sterile filtered PBS per condition and transferred onto slides using the Shandon Cytospin 4 (ThermoFisher Scientific, Waltham, MA, USA). Cells were not permeabilized prior to staining, in order to specifically evaluate presence of CXCR4 at the plasma membrane. Slides were air dried for 5 minutes, washed in PBS with 0.5\% BSA (PBSA), and either incubated in primary antibody against CXCR4, diluted 1:100 in PBSA (Abcam, Cambridge, UK), or PBSA for $1 \mathrm{hr}$ at $37^{\circ} \mathrm{C}$ in the dark. Samples were washed once in PBSA and incubated in secondary antibody anti-rabbit IgG Alexa Fluor 488, diluted 1:800 (ThermoFisher Scientific, Waltham, MA, USA), for $1 \mathrm{hr}$ at $37^{\circ} \mathrm{C}$ in the dark. Samples were washed in PBSA and stained with $0.5 \mathrm{ug} / \mathrm{mL}$ DAPI for 5 minutes in the dark and washed once with PBSA. Slides were imaged using Zeiss Imager.Z2.

\subsection{Western Blot}

Total cellular protein was extracted from $1 \times 10^{6}$ cells by boiling in $70 \mathrm{uL}$ of $1 \mathrm{X}$ protein loading buffer for 15 minutes. Protein samples were resolved in a $10 \%$ SDS gel at $140 \mathrm{~V}$ for $1.15 \mathrm{hr}$, and 
the resolved proteins were transferred to a nitrocellulose membrane at $100 \mathrm{~V}$ for $2 \mathrm{hr}$. Membranes were blocked in 5\% milk in Tris-Buffered Saline (TBS) for $1 \mathrm{hr}$, then incubated overnight at $4^{\circ} \mathrm{C}$ in primary antibody against CXCR4 (Abcam Cambridge, UK) or CALM (Sigma, St. Louis, MI, USA), diluted 1:1000 in TBS containing $0.1 \%$ TWEEN. Following primary antibody incubation, membranes were washed $3 \mathrm{x}$ in TBST for 5 minutes and incubated with HRP-conjugated anti-rabbit antibodies $(1: 10,000)$ for 1 hour. After washing as above, membranes were developed with BioRad Molecular Imager ChemDoc XRS+ system using a Clarity Western ECL substrate kit (BioRad, Hercules, CA, USA). To confirm equal loading of proteins across the experimental conditions, membranes were subsequently incubated with a primary antibody against $\beta$-actin at 1:1,000 (Cell Signaling Technologies, Danvers, MA, USA), developed, and imaged as described above.

For co-culture experiments, $1.5 \times 10^{5} / \mathrm{mL}$ hMSC cells were plated on $10 \mathrm{~cm}$ plates and incubated at $37^{\circ} \mathrm{C}$ with $5 \% \mathrm{CO}_{2}$ for 24hrs. $4.5 \times 10^{5} / \mathrm{mL}$ U937 cells were added to previously cultured hMSCs and incubated at $37^{\circ} \mathrm{C}$ with $5 \% \mathrm{CO}_{2}$ for $24 \mathrm{hrs}$. Following the $24 \mathrm{hr}$ incubation, co-cultured cells were exposed to $1 \mathrm{uM}$ of AMD3465, a CXCR4 inhibitor (Selleck Chemicals, Houston, TX, USA), for 4 hrs. Suspension U937 cells were harvested at $1 \times 10^{6}$ / $70 \mathrm{uL}$ of $1 \mathrm{X}$ loading buffer and boiled for 15 minutes. Samples were loaded on $15 \%$ SDS gel, run at $140 \mathrm{~V}$ for $3.36 \mathrm{hr}$, and transferred to a nitrocellulose membranes at $100 \mathrm{~V}$ for $2 \mathrm{hr}$. Five percent BSA in Tris-Buffered Saline (TBS) was used to block membranes for $1 \mathrm{hr}$. Membranes were incubated in primary antibody solutions with TBS containing 0.1\% TWEEN for $\mathrm{p} 44 /$ 42 (ERK) at 1:1,000 (Cell Signaling Technologies, Danvers, MA, USA) and phospho-p44/42 (phospho-ERK) at 1:2000 (Cell Signaling Technologies, Danvers, MA, USA) overnight at $4^{\circ} \mathrm{C}$. Membranes were developed and imaged as stated above, and subsequently incubated in $\beta$-actin as described above.

All western blots are done in duplicate with one representative experiment shown.

\subsection{Real-Time qPCR}

Cells $\left(1 \times 10^{6}\right)$ were harvested in $300 \mathrm{uL}$ of Trizol reagent and RNA was extracted using Directzol RNA MiniPrep Plus kit (Zymo Research, Irvine, CA, USA) and quantified using the Thermo Scientific Nanodrop 2000. cDNA was synthesized using SuperScript III Reverse Transcriptase kit (Fisher Scientific, Waltham, MA, USA). Real-time PCR was performed on a ViiA 7 Real-time PCR with the ITaq Universal SYBR Green Supermix and probes for: human GAPDH forward (5' ACCCACTCCTCCACCTTTGAC 3'), reverse (5' TGTTG CTGTAGCCAAATTCGTT 3'); CXCR4 forward (5' CACTTCAGATAACTACACCG 3'), and reverse (5' ATCCAGACGCCAACATAGAC 3'); CXCL12 forward (5' GGACTTTCCGCTAGACCCAC 3'), and reverse (5' GTCCTCATGGTTAAGGCCCC 3’).

\subsection{Scratch Assay}

One million MEFs were plated in a 6 well tissue culture plate 24 hours prior to the experiment, allowing growth to confluence. At hour 24 , a single scratch was made using a $200 \mathrm{uL}$ pipette tip. The media was aspirated off, each well was gently rinsed with PBS, and $2 \mathrm{~mL}$ fresh media slowly added. Cells were photographed at hours 0,4 , and 8 . Given the short time course of this experiment, anti-mitotic agents were not used. Photographs were processed by ImageJ to measure the area of the scratch at each time point.

\subsection{Transwell Migration Assay}

Corning transwell plates $(8 \mu \mathrm{m}$ pore size, Fisher Scientific, Waltham, MA, USA) were used to assess migration. CXCL12 (R\&D Systems, Minneapolis, MA, USA), or control (PBS), was added at $100 \mathrm{ng} / \mathrm{uL}$ to $1.5 \mathrm{x} 10^{5}$ Kasumi- 1 and U937 cells and incubated for $24 \mathrm{hrs}$ at $37^{\circ} \mathrm{C}$ with $5 \% \mathrm{CO}_{2}$. After $24 \mathrm{hrs}$, inserts were removed, each well was agitated via pipette, and cells were incubated for 30 minutes prior to imaging. Three images were taken of each corresponding well and representative areas of each image were analyzed via ImageJ (23). Two independent investigators performed each analysis to assure reproducibility. The above was repeated in U937 cells following a $4 \mathrm{hr}$ incubation in $0.25 \mathrm{uM}, 0.5 \mathrm{uM}$, or $1 \mathrm{uM}$ AMD3465 (Selleck Chemicals, Houston, TX, USA) or control (water). Following a $4 \mathrm{hr}, 8 \mathrm{hr}$, and $24 \mathrm{hr}$ incubation in transwell plates, images were taken and analyzed as detailed above.

\subsection{Cytotoxicity Assays}

\subsubsection{Flow Cytometry}

Human MSC cells were plated at $1 \times 10^{5}$ cells $/ \mathrm{mL}$ per well of a 6well plate. Cells were cultured for 8 hrs, then $3 \times 10^{5} / \mathrm{mL}$ U937 cells were added to the hMSCs culture. Co-cultures were maintained for $16 \mathrm{hrs}$ at $37^{\circ} \mathrm{C}$ with $5 \% \mathrm{CO}_{2}$. Twenty-four hours from initial hMSC plating, Doxorubicin (1uM) and AMD3465 (1uM) (Selleck Chemicals, Houston, TX, USA) were added to cocultured cells and allowed to incubate $37^{\circ} \mathrm{C}$ with $5 \% \mathrm{CO}_{2}$ for $24 \mathrm{hrs}$. Suspension cells were removed from wells and combined with corresponding adherent cells trypsinized with $0.25 \%$ trypsin. Samples were washed once in phenol free RPMI 1640 media. Non-specific binding was blocked with normal mouse IgG 1:200 (Santa Cruz Biotechnology, Dallas, TX, USA) in 1\% BSA (50uL per $1 \times 10^{6}$ cells) for 30 minutes in the dark at $4^{\circ} \mathrm{C}$. Primary antibody APC-conjugated mouse, anti-human CD45 (BD Pharmingen, San Diego, CA, USA) was added to cell solutions at 1:10, and incubated on ice for 30 minutes in the dark. Cells were washed once with phenol free RPMI 1640 and resuspended in 1uM DAPI solution. Samples were run on BD LSRII and analyzed using FlowJo software.

\subsubsection{BrdU Assay}

Cells were plated at $5 \times 10^{5}$ cells $/ \mathrm{mL}$ media and treated with one of the following conditions: vehicle control (sterile water), $1 \mathrm{uM}$ AMD3465, 2uM AMD3465, 2.5uM cytarabine, 100nM cytarabine, 1uM AMD3465 + 2.5uM cytarabine, $1 \mathrm{uM}$ AMD3465 followed four hours later by $2.5 \mathrm{uM}$ cytarabine, $1 \mathrm{uM}$ AMD3465 + 100nM cytarabine, 1uM AMD3465 followed four hours later by $100 \mathrm{nM}$ cytarabine, 2uM AMD3465 + 100nM cytarabine, and 2uM AMD3465 followed four hours later by $100 \mathrm{nM}$ cytarabine. Treated cells were incubated at 37 degrees with 5\% CO2 for 48 hours and were then analyzed by BrdU staining (Cell Signaling Technology, Danvers, MA, USA). 
Absorbance was read at 450nM on a Perkin Elmer multimode plate reader.

\subsection{Statistical Analysis}

All data are expressed as the mean \pm Standard Error of the Mean (SEM), unless otherwise noted. Differences between two experimental groups were analyzed by unpaired t-test. Ordinary one-way ANOVA was used to compare differences between multiple groups. A p-value $\leq 0.05$ was considered significant.

\section{RESULTS}

\subsection{Enhanced Cell Migration in CALM-AF10 Transformed Cells}

Murine embryonic fibroblasts (MEFs) were stably infected with CALM-AF10 or an empty vector (Figure 1A), and scratch assays were performed to assess the impact of the CALM-AF10 fusion oncoprotein on cell migration. Cells were plated the day prior to achieve confluence at the time of scratch. Closure of the wound was examined at four and eight hours post scratch infliction, and percent wound closure calculated. The CALM-AF10 transformed MEFs showed a 35 percent wound closure at 4 hours, compared to $19 \%$ closure in the empty vector cells $(\mathrm{p}<0.05)$. This difference was magnified at the 8 -hour time point, with CALM-AF10 transformed cells showing a $85 \%$ closure, compared with $61 \%$ in the empty vector cells ( $\mathrm{p}=0.04)$ (Figures 1B, C).

We then examined the migratory behavior of human leukemia cell lines and sought to identify cytokines that could be involved in the migration of CALM-AF10 leukemias. Because CXCL12 is the primary ligand for the CXCR4 receptor, we investigated the migratory behavior of CALM-AF10 translocated leukemias when treated with CXCL12. We found that CALM-AF10 translocated U937 cells demonstrated an increase in cell migration upon stimulation with CXCL12, whereas the CALM-AF10 negative Kasumi-1 cells did not have a significant change in cell migration with the addition of CXCL12. U937 cells exhibited a nearly 3-fold increase in migration compared to Kasumi-1 cells when treated with $100 \mathrm{ng} / \mathrm{mL}$ CXCL12 over a $24 \mathrm{hr}$ period (Figures 1D, E, n=3, $\mathrm{p}<0.001)$. CXCL12 is measured in U937 and Kasumil cells by qPCR and reveals that CXCL12 is not expressed in either cell line at any significant level (Figure 1F: CXCR4 expression is shown as a reference). When analyzing the effects of a CXCR4 inhibitor, we found that AMD3465 treatment decreased the migration of CALM-AF10 translocated leukemias. Following a $4 \mathrm{hr}$ treatment with 1uM AMD3465, U937 cells treated with $100 \mathrm{ng} / \mathrm{mL}$ of CXCL12 for 24 hours had a 6-fold decrease in migration compared to cells exposed to vehicle control (Figure 1G, $n=3$, $\mathrm{p}<0.01$ ). Dose and time response curves with and without CXCL12 as a chemoattractant reveal migration of U937 cells over time (normalized to the 0 AMD3465 condition) is minimally impacted by increasing concentrations of AMD3465 in the absence of CXCL12. There is a trend toward diminished migration with low doses of AMD3465 in the presence of CXCL12 (Figure 1H). Time course experiments reveal a trend toward a greater impact of CXCL12 with short exposures to chemoattractant (Figure 1I: 0uM AMD3465 at 4 hour vs 24 hour, $\mathrm{p}=0.06$ ). These observations indicate that AMD3465 exerts an inhibitory effect on migration of CALM-AF10 expressing leukemia cells.

\subsection{Increased Expression of CXCR4 in CALM-AF10 Leukemia}

We assessed CXCR4 expression in U937 and Fujioka, two myeloid leukemia cell lines containing the CALM-AF10 translocation, and compared it with that in Kasumi-1, a myeloid leukemia cell line that does not express CALM-AF10. The mRNA expression of CXCR4 in the U937 and Fujioka cells was 100 -fold greater when compared with the Kasumi-1 cells (Figure 2A, $\mathrm{p}<0.001$ ). Consistent with the elevated transcript levels, total CXCR4 protein was also increased in CALM-AF10translocated cell lines (Figure 2B). Immunofluorescence microscopy further revealed that CXCR4 was primarily localized at the plasma membrane, to a greater degree in CALM-AF10 translocated leukemias (Figure 2C).

We determined the CXCR4 expression in secondary murine cell line models, characterized by either CALM-AF10 or Hoxa9Meis1 chromosomal translocations, to further validate the relationship between CALM-AF10 and increased CXCR4 expression in human AML cell lines. In concordance with our findings in the human cell line models, we found an increase in total protein expression and plasma membrane receptor expression in the murine CALM-AF10 leukemia cells relative to the Hoxa9-Meis1 translocated cells (Figures 2D, E).

\subsection{Effect of CXCR4 Inhibition in Combination With Doxorubicin}

We next examined the potential of CXCR4 as a therapeutic target in CALM-AF10-driven leukemia. We co-cultured the CALMAF10 expressing U937 cell line with human mesenchymal stem cells (hMSCs) to model the interaction between the bone marrow microenvironment and leukemia cells. With CD45 as a marker for leukemia cells, we employed dual color flow cytometry to separate and analyze the effect of targeted and cytotoxic treatments on leukemia cells in co-culture. We utilized this system to investigate the possible synergistic effect of AMD3465, a CXCR4 inhibitor, in combination with doxorubicin, a standard chemotherapeutic agent. We first assessed the ability of AMD3465 to inhibit CXCR4 activation by examining its effect on the MAPK pathway, a known downstream effector of CXCR4 signaling. U937 cells were cultured with hMSCs for $24 \mathrm{hrs}$ before treatment with $1 \mathrm{uM}$ of AMD3465 for 4hrs. hMSCs are known to express the ligand for CXCR4, CXCL12. We confirmed expression of CXCL12, as well as lack of CXCR4 expression, in our hMSCs via qPCR (Figure 3A). Western blot analysis revealed a decrease specifically in the phosphorylated ERK after AMD3465 treatment (Figure 3B), confirming an active CXCR4 signaling cascade in CALM-AF10 expressing leukemia cells. We then examined the impact of AMD3465 on activation of the ERK pathway on U937 cells that were cultured in charcoal stripped, 
A

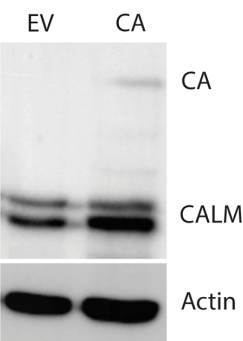

D

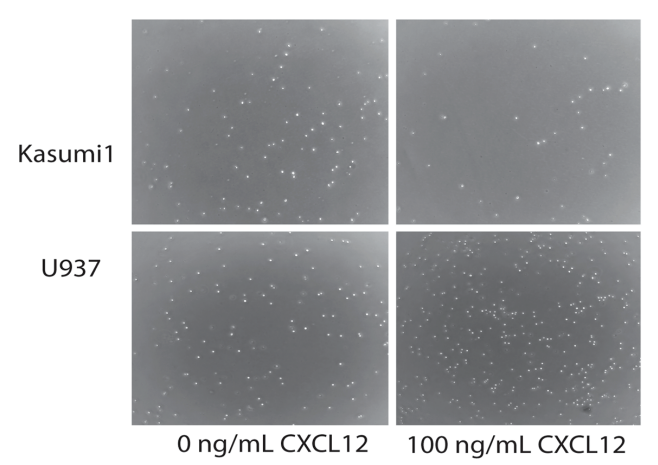

H
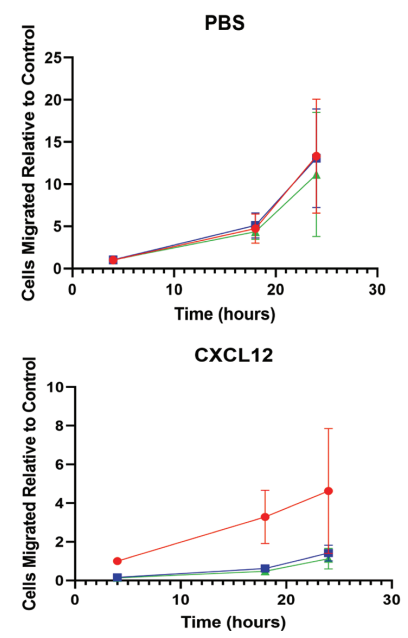

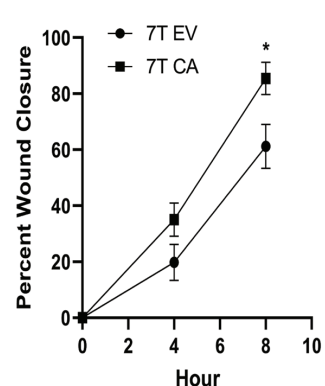

Hour
- OUM AMD3465

- $0.25 \mathrm{uM}$ AMD3465

- $0.5 \mathrm{LMM}$ AMD3465

- 0 AMD3465

-0.25 AMD3465

$₫ \quad 0.5$ AMD3465
C

Oh

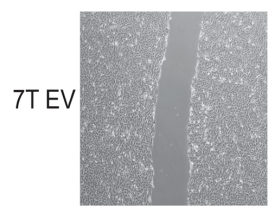

$7 \mathrm{TCA}$

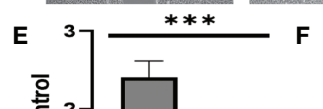

$4 h$
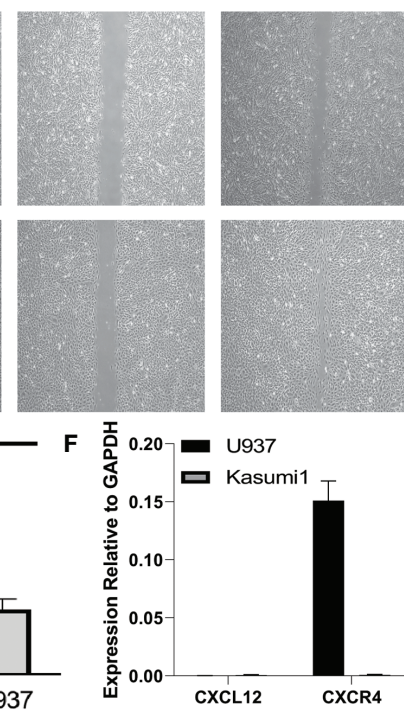

G

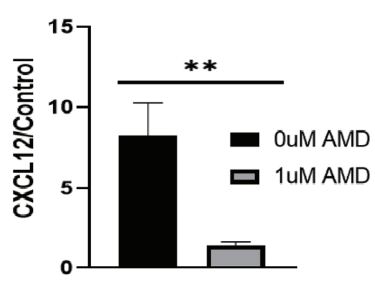

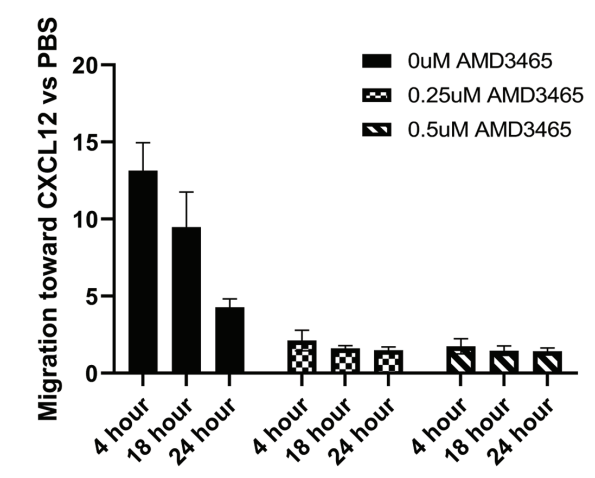

FIGURE 1 | CALM-AF10 leukemia cells show enhanced migration, influenced by CXCL12. (A) A Western blot of MEFs transformed by empty vector (EV) or CALM-AF10 (CA) confirms the presence of the CA oncoprotein. (B) Accelerated wound closure in murine embryonic fibroblasts (MEFs) transformed by CALM-AF10. (C) Representative images from one replicate of scratch assay demonstrate more rapid wound closure in CALM-AF10 transduced MEFs. (D) Representative images from one transwell assay are shown. (E) The impact of CXCL12 as a stimulant for cell migration is examined in U937 and Kasumi cells. (F) qPCR of CXCL12 and CXCR4 in U937 and Kasumi1.

(G) The impact of the CXCR4 inhibitor AMD3465 on the migration of U937 cells is evaluated. (H) Dose and time response curves of U937 cells exposed to AMD3465 with and without CXCL12 stimulation reveal migration U937 cells over time (normalized to the 0 AMD3465 condition). (I) Dose and time response experiments in U937 cells using AMD3465 in the present or absence of CXCL12 as a chemoattractant. ${ }^{*} \mathrm{p}<0.05 ;{ }^{* \star} \mathrm{p}<0.01 ;{ }^{\star \star *} \mathrm{p}<0.001$.

phenol-free RPMI and treated with CXCL12 in order to further examine the impact of specific CXCR4 inhibition. Western blot analysis again revealed a decrease in pERK/ERK in the AMD3465 treated cells (Figure 3C). Total ERK levels were used as a control and remained unchanged.
We next sought to evaluate the cytotoxic effect of CXCR4 inhibition. Following $16 \mathrm{hrs}$ of co-culture, cells were exposed to either 1uM AMD3465, 1uM doxorubicin, or the combination for $24 \mathrm{hrs}$ and analyzed for cell viability using flow cytometry. One micromolar dosing of doxorubicin is known to be cytotoxic to 
A

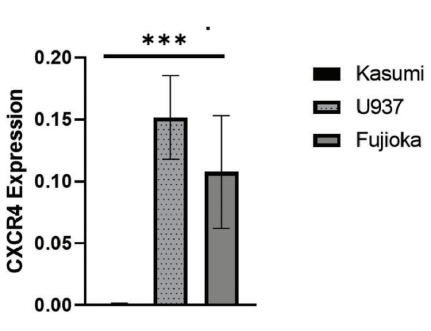

C

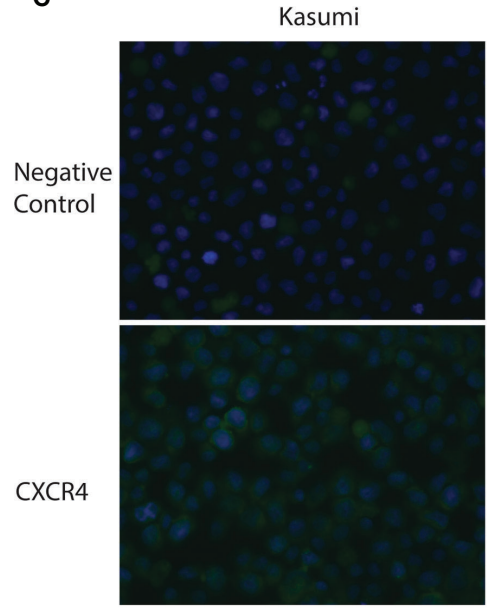

D
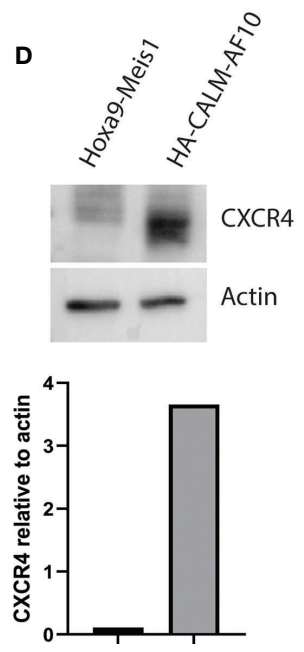

- HM30.6

ㅁ HACA66.11
B
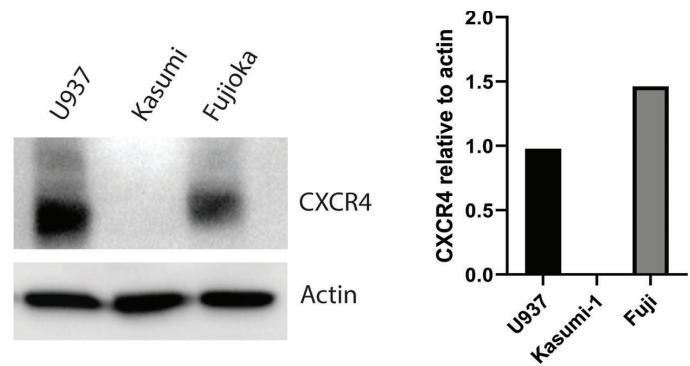

U937
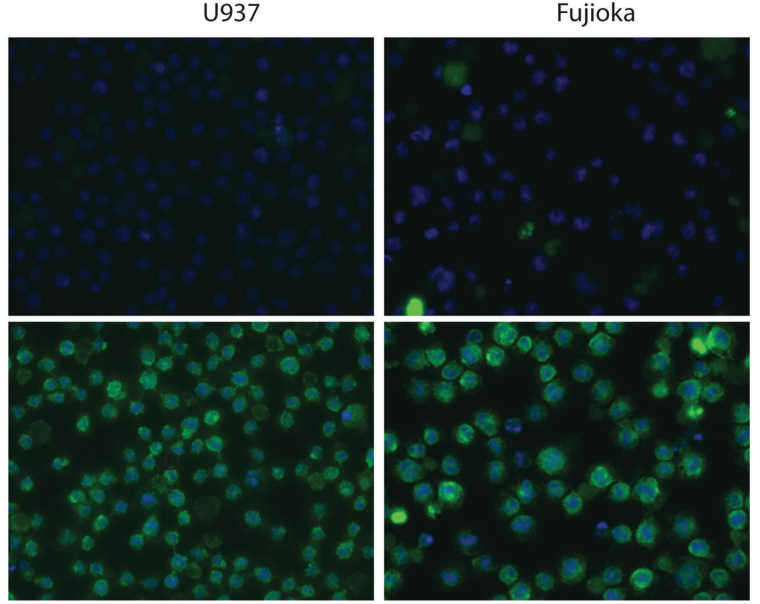

E

Negative Control
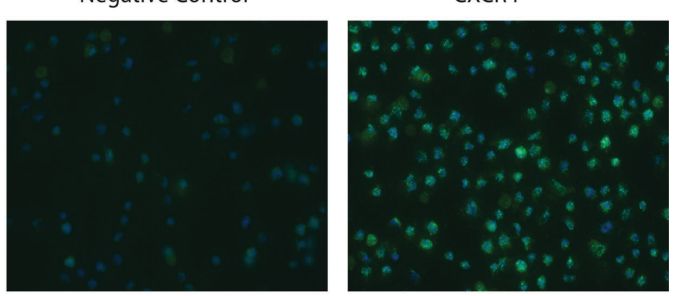

HA-CALM-AF10
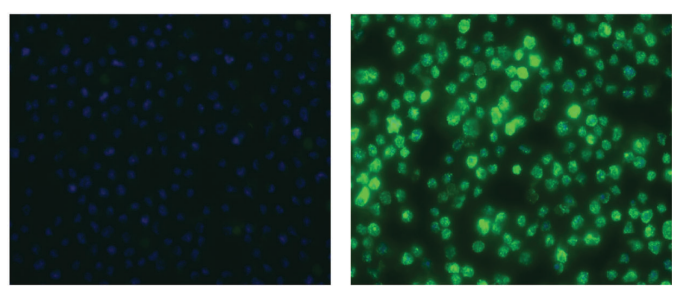

FIGURE 2 | Increased CXCR4 expression in CALM-AF10 transformed leukemia cells. CXCR4 expression is evaluated in human myeloid leukemia cell lines characterized by the CALM-AF10 translocation (U937 and Fujioka) and compared with a myeloid leukemia cell line not containing the CALM-AF10 translocation. Expression is examined by transcript quantification, with values shown relative to GAPDH (A), and protein evaluation by Western blot, with corresponding densitometry normalized to actin (B). Immunofluorescence staining of human leukemia cells reveals increased CXCR4 expression is concentrated on the plasma membrane (C). (D) High CXCR4 protein expression is confirmed by Western blot in a murine leukemia induced by CALM-AF10, compared with a murine leukemia characterized by the Hoxa9-Meis1 translocation, with corresponding densitometry shown, normalized to actin. (E) Immunofluorescence staining in the CALM-AF10 translocated murine leukemia cells reveals increased CXCR4 expression is concentrated on the plasma membrane. ${ }^{\star \star \star} p<0.001$.

U937 cells and this was confirmed in our lab (24), and data not shown). We found that the AMD3465 treatment alone did not have a significant cytotoxic effect; the control and AMD3465 treated cells showed 89.53 percent and 90.04 percent cell viability, respectively $(\mathrm{n}=4)$. We also did not find a significant difference in cell viability between the AMD3465 in combination with doxorubicin (75.63 percent) and doxorubicin alone (75.33 percent) (Figures 3D, E).

We then investigated the potential anti-proliferative effect of CXCR4 inhibition alone or in combination with traditional 


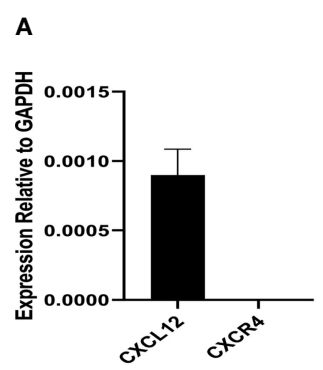

D
B

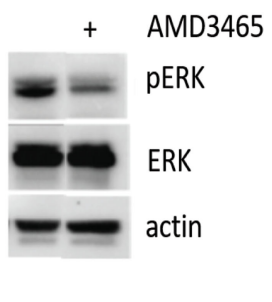

C

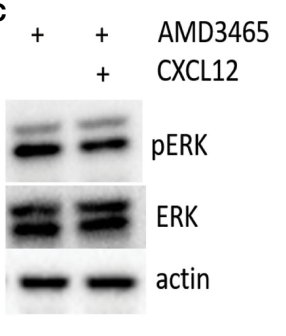

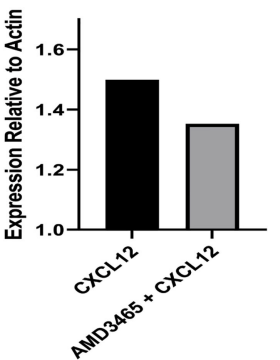

E
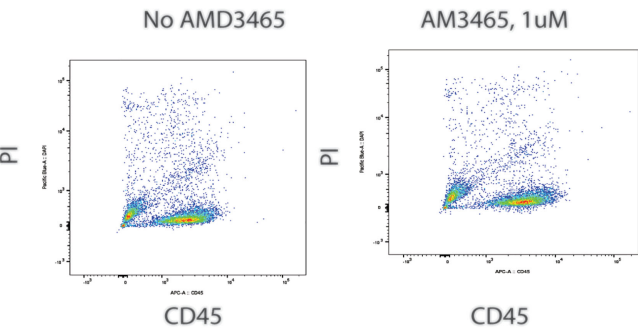

CD45

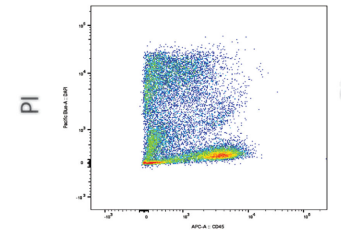

CD45

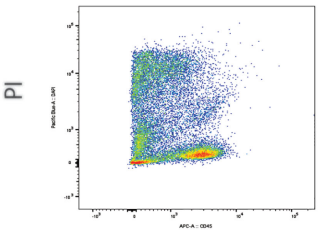

CD45
F

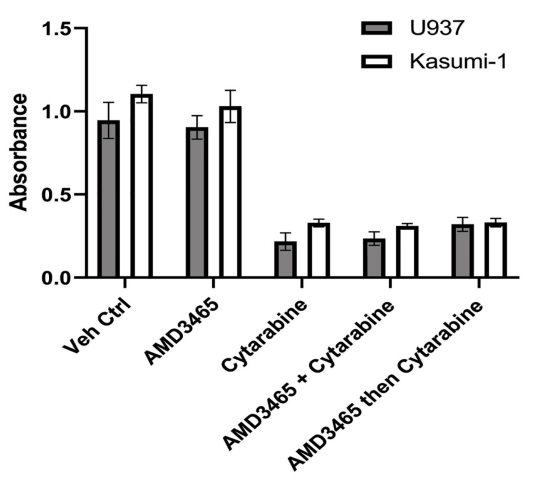

G

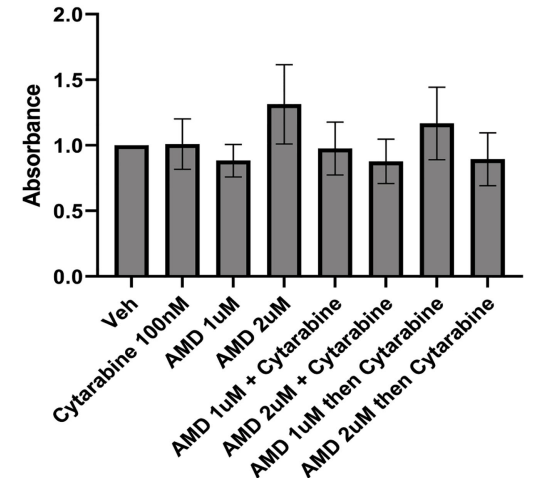

FIGURE 3 | Effect of CXCR4 inhibition on cell proliferation. U937 cells are kept in co-culture with human mesenchymal stem cells (hMSCs) and the cytotoxic effect of a CXCR4 inhibitor, AMD3465 is examined. (A) The expression of CXCL12 and CXCR4 in hMSCs is shown, normalized to GAPDH. (B) AMD3465 produces a decrease in pERK/ERK in U937 cells kept in co-culture in regular media. (C) The impact of AMD3465 on pERK/ERK in U937 cells kept in co-culture in charcoal stripped, phenol-free media in the presence of CXCL12 is shown by western blot, with corresponding densitometry. (D) The cytotoxic effect of AMD3465 is examined, both with and without doxorubicin. (E) Representative FACS data is shown. Propidium iodide (PI) staining delineates live versus dead cells, and CD45 positivity separates the CD45+ leukemia cells from the CD45- hMSCs. (F) The anti-proliferative effect of CXCR4 inhibition is evaluated by BrdU assay, with and without treatment dose cytarabine. (G) The anti-proliferative effect of high dose and low dose CXCR4 inhibition is evaluated by BrdU assay, with and without subtoxic dose cytarabine.

chemotherapy. Cells were treated with either 1uM AMD3465, $2.5 \mathrm{uM}$ cytarabine, AMD3465 and cytarabine together, or AMD3465 followed by cytarabine 4 hours later. This dose of cytarabine has previously been reported to be cytotoxic to U937 cells, and we found a 77\% reduction in U937 cell proliferation with 2.5uM cytarabine (25). Human leukemia cells lines U937 (CALM$A F 10+)$ or Kasumi-1 (CALM-AF10 negative) were treated for 48 hours, and cell proliferation was quantified via BrdU assay. The 
cytarabine treatment produced the expected anti-proliferative effect with a 70-80\% decrease in absorbance in both cell lines examined. However, AMD3465 showed no anti-proliferative effect in either cell line. This was true when cells were treated with AMD3465 alone, and when AMD3465 was used in combination with cytarabine, either simultaneously or sequentially (Figure 3F). We then performed additional cell proliferation assays with low dose cytarabine $(100 \mathrm{nM})$, to evaluate whether a subtoxic dose of cytarabine would uncover a potential therapeutic effect of AMD3465. These experiments additionally incorporated AMD3465 at $2 \mathrm{uM}$ to assess the potential therapeutic impact of a higher dose of the CXCR4 inhibitor. We found no additive nor synergistic effect of AMD3465 at 1uM or at $2 \mathrm{uM}$ in combination with subtoxic doses of cytarabine, when used simultaneously or sequentially (Figure 3G).

\section{DISCUSSION}

\subsection{CALM-AF10 Transformed Cells Exhibit Enhanced Cell Migration}

The $t(10 ; 11)(p 13 ; q 14-21)$ chromosomal translocation encodes the leukemogenic CALM-AF10 fusion oncoprotein. The CALM-AF10 translocation is found in both acute myeloid and acute lymphoid leukemias and are found most frequently in $\mathrm{T}$ ALL at an incidence of about 15\%. CALM-AF10 leukemia is driven by overexpression of HOX homeobox cluster genes (26, 27). The upregulation of HOXA genes in CALM-AF10 leukemia is dependent on AF10's recruitment of DOT1L, and resultant focal H3K79 hypermethylation specifically at the HOXA locus (8, 9). CALM-AF10 also interacts with the CRM1/XPO1 nuclear export receptor via CALM's nuclear export signal peptide sequence, which is retained in the fusion protein, and is necessary for leukemogenesis. This interaction is required for localization of CALM-AF10 to the HOXA cluster, and for resultant HOXA overexpression (3). The PHD1-zinc-knucklePHD2 (PZP) domain of AF10 is consistently interrupted in $C A L M-A F 10$ translocations. It has been recently identified that the disruption of the PZP domain of AF10 disrupts the normal localization of DOT1L across the genome, allowing DOT1L to be tethered to the HOXA locus by CALM-AF10 (28).

$C A L M-A F 10$ leukemias typically show a poor response to therapy, have an increased propensity to relapse and have a worse overall prognosis. The poor clinical outcome of CALMAF10-driven leukemia necessitates the need for better understanding of how the translocation affects the behavior of the disease so that novel targeted therapies may be developed. Interestingly, $C A L M-A F 10$ leukemias have a high prevalence of extramedullary disease, including bulky mediastinal disease, and a propensity for central nervous system relapse. Emerging evidence reveals a role for factors influencing cell adhesion and migration in these processes. While these processes may be separate from the fundamental mechanisms of tumorigenesis, they are potentially critical to tumor development, metastasis and chemotherapeutic response.
We therefore examined the migratory properties of cells transformed by $C A L M-A F 10$ and found that MEFs transformed by CALM-AF10 exhibited enhanced cell migration in scratch assays, compared with controls. We then examined cell migration in CALM-AF10+ leukemia cells via transwell migration assays and discovered that CALM-AF10+ U937 cells show enhanced migration in transwell assays, compared with CALM-AF10 negative Kasumi-1 cells. Furthermore, the U937 cells showed a robust increase in cell migration toward a CXCL12 stimulus; whereas the presence of CXCL12 did not influence Kasumi-1 cell migration. Neither leukemia cell line expresses significant amounts of CXCL12, thus CXCL12 secretion and autocrine signaling are not impacting this differential response.

\subsection{Increased CXCR4 Expression Is Seen in CALM-AF10 Leukemia and Contributes to Cell Migration}

CXCL12 is the primary ligand for the chemokine receptor CXCR4, found on cell surface of HSCs and some leukemia cells. The interaction between CXCR4 on HSCs, and its ligand CXCL12, leads to HSC migration and subsequent homing to the bone marrow (29). Evidence suggests that CXCR4 plays a role in leukemia cell migration as well, and one study correlated increased CXCR4 expression on lymphoblasts with a trend toward bulky extramedullary disease in pediatric ALL (22). We investigated whether the increased cell migration we observed in CALM-AF10 transformed cells correlated with CXCR4 expression.

Following the discovery that CALM-AF10 expressing leukemia cells migrate toward a CXCL12 stimulus, we tested whether blocking this interaction could halt migration. We found that the CXCR4 inhibitor AMD3465 abrogated this migration, confirming that CXCR4 was the integral mediator of this effect. Consistent with this finding, we found an increase in CXCR4 expression specifically in human leukemia cell lines carrying the CALM-AF10 translocation. This finding was noted at both the transcript and protein levels. To ensure broader validity of these observations, we also confirmed our findings in murine leukemia cell lines previously generated in our lab.

CXCL12 is not only present in the bone marrow but is found in every tissue in the body, except in the CNS. CXCL12 is highly expressed in other lymphoid tissues such as the thymus, lymph nodes and spleen. It is also expressed in the liver and testes. These are all sites of potential leukemic involvement, with extramedullary involvement being characteristic of CALMAF10 leukemias (30). It is possible that high CXCR4 expression on the plasma membrane of CALM-AF10 leukemias contributes to the development of extramedullary disease. Additional in vivo studies are needed to definitively establish the relationship between CXCR4 expression and extramedullary disease development in CALM-AF10 leukemia.

\subsection{The Effect of CXCR4 Inhibition on CALM-AF10 Leukemia Cells}

Based on our findings of increased CXCR4 expression on CALM-AF10 leukemic blasts, and the contribution of CXCR4 
to $C A L M-A F 10+$ cell migration, we hypothesized that these cells would be particularly sensitive to CXCR4 inhibition as a chemosensitizer. We first established that CALM-AF10 expressing leukemia cells have an active CXCR4 signaling cascade by observing a reduction in phophoERK to ERK after CXCR4 inhibition. We then sought to examine the potential of CXCR4 inhibition to contribute to either cytostatic or cytotoxic chemotherapy effects.

Cytarabine is commonly used in the treatment of both ALL and AML. It is a pyrimidine analog and competes with cytidine for incorporation into DNA during repair and replication. When cytarabine is incorporated into DNA, it halts replication, exerting a cytostatic effect. Intriguingly, despite an intact CXCR4 signaling cascade, we found no decrease in cell proliferation upon exposure of U937 cells to AMD3465 in combination with either subtoxic or therapeutic concentrations of cytarabine.

Doxorubicin is another chemotherapy agent commonly used for the treatment of both ALL and AML. It is a cytotoxic agent, intercalating with DNA and causing double strand DNA breaks. Doxorubicin also inhibits topoisomerase II and results in apoptosis. We studied the effects of doxorubicin in combination with AMD3465 in U937 cells, and surprisingly found no synergistic nor additive effect of the combination.

Binding of CXCR4 by its ligand may result in activation of a multitude of signaling cascades, including those involved in cell survival and proliferation, such as the PI3K and ERK signaling pathways. CXCR4 inhibition is therefore an attractive target as a chemotherapeutic adjunct. CXCR4 inhibition has been examined in acute leukemia, both as a potential therapy and as a stem cell mobilizer. Previous studies have demonstrated decreased cell proliferation in AML cells in vitro, and decreased AML engraftment in vivo with the use of CXCR4 inhibitors $(31,32)$. A prior study reported that lymphoblastic leukemia cells in co-culture were sensitized to vincristine by a CXCR4 inhibitor through the upregulation of Bax (33). However, there are conflicting data in the published literature. For example, a 2013 study revealed that CXCL12 stimulation resulted in increased apoptosis in AML cell lines, mediated by $\mathrm{Bcl} 2$ family members (34). In addition, an examination of childhood ALL patient samples revealed variability in chemotactic and proliferative response to CXCR4 inhibition (35).

The factors contributing to these variable and sometimes conflicting findings are not well elucidated. CXCR4 expression and signaling is regulated through a variety of mechanisms. At a transcriptional level, CXCR4 expression may be modified through DNA methylation, directly activated by multiple transcription factors, and impacted by a range of physiologic stimuli $(19,36)$. Protein expression may be impacted by tyrosine sulfation or glycosylation or modified by oligomerization. There are multiple processes which separately regulate CXCR4 signaling once bound by ligand, impacting receptor internalization, degradation, and recycling (19).

Ultimately, CXCR4 expression on leukemic blasts varies, and the factors influencing differences in expression, function, and downstream signaling in leukemia are unknown. It is also not known whether degree of CXCR4 expression influences therapeutic response to CXCR4 inhibition. Herein, we identify a genetically defined a subtype of leukemia, which is characterized by high CXCR4 expression and has an intact CXCR4 signaling pathway. We report the novel findings that despite an intact CXCR4 signaling pathway, CALM-AF10 carrying leukemia cells do not show additive nor synergistic cytotoxic or anti-proliferative effects upon combinatorial inhibition of CXCR4 and traditional chemotherapeutics.

Jost et al. present a model of CNS meningeal infiltration by T-lymphoblasts through CXCR4 mediated bone marrow colonization (37). Others have provided evidence that CXCR4 inhibition prevented homing of multiple myeloma cells to the bone marrow compartment (38). It is possible the use of CXCR4 inhibitors in specific clinicopathologic scenarios may yet provide clinical benefit. The studies presented here are limited by the use of cell lines, and future studies examining CXCR4 expression and the use of CXCR4 inhibitors in patient samples will add valuable insight to the biology of CALM-AF10 leukemia. Additional studies are needed to establish whether CXCR4 inhibition impacts other characteristics of CALM-AF10 leukemia, including $\mathrm{CNS}$ invasion. We utilized a co-culture system to simulate the bone marrow microenvironment; however, additional insight would be gleaned utilizing three dimensional bone marrow scaffolds or in vivo models of disease $(39,40)$.

\subsection{Summary}

Due to its status as a G-protein coupled receptor, CXCR4 plays a role in many downstream pathways controlling cell adhesion, migration, survival and proliferation. Previous studies have reported therapeutic effect of CXCR4 inhibition in some acute leukemias in the preclinical setting, as described above. However, clinical trials of CXCR4 inhibitors have not shown promising results, particularly in ALL (41-43). Specific factors that determine response to CXCR4 inhibition have not been discovered. In this study, we demonstrate that CALM-AF10 translocated leukemias show an increased expression of CXCR4, as well as an increase in CXCL12-stimulated cell migration. We posited that this increase would lead to an enhanced sensitivity to CXCR4 inhibitors. However, we observed no decrease in cell proliferation nor cytotoxic effect with CXCR4 inhibition. We have therefore identified a subtype of leukemia that is not sensitive to anti-proliferative or cytotoxic effect CXCR4 inhibitors, despite high levels of CXCR4 expression and an intact CXCR4 signaling pathway. This finding strongly suggests caution moving forward with CXCR4 inhibition as a therapeutic adjunct. By elucidating factors that influence response to CXCR4 inhibition, these targeted therapeutics can be better tailored to specific diseases.

\section{DATA AVAILABILITY STATEMENT}

The original contributions presented in the study are included in the article/supplementary material. Further inquiries can be directed to the corresponding author. 


\section{AUTHOR CONTRIBUTIONS}

$\mathrm{SF}$ and $\mathrm{JH}$ performed the experiments. SF and $\mathrm{JH}$ wrote the paper. SZ, GS, and JS provided input into experimental design and analysis, and critically reviewed the manuscript. All authors contributed to the article and approved the submitted version.

\section{FUNDING}

This work was supported by a contribution from Donna and Martin Waldron (JH). The research reported here was supported by grant U54 GM115516 from the National Institutes of Health for the Northern New England Clinical and Translational Research network (GS, JH). This work was also supported in part by the Emily M. Lyman Pediatric Leukemia Research Fund

\section{REFERENCES}

1. Tebar F, Bohlander SK, Sorkin A. Clathrin Assembly Lymphoid Myeloid Leukemia (CALM) Protein: Localization in Endocytic-Coated Pits, Interactions With Clathrin, and the Impact of Overexpression on Clathrin-Mediated Traffic. Mol Biol Cell (1999) 10(8):2687-702. doi: 10.1091/mbc.10.8.2687

2. Scotland PB, Heath JL, Conway AE, Porter NB, Armstrong MB, Walker JA, et al. The PICALM Protein Plays a Key Role in Iron Homeostasis and Cell Proliferation. PloS One (2012) 7(8):e44252. doi: 10.1371/journal.pone.0044252

3. Conway AE, Scotland PB, Lavau CP, Wechsler DS. A CALM-Derived Nuclear Export Signal is Essential for CALM-AF10-Mediated Leukemogenesis. Blood (2013) 121(23):4758-68. doi: 10.1182/blood-2012-06-435792

4. Aasland R, Gibson TJ, Stewart AF. The PHD Finger: Implications for Chromatin-Mediated Transcriptional Regulation. Trends Biochem Sci (1995) 20(2):56-9. doi: 10.1016/S0968-0004(00)88957-4

5. Aravind L, Landsman D. AT-Hook Motifs Identified in a Wide Variety of DNA-Binding Proteins. Nucleic Acids Res (1998) 26(19):4413-21. doi: $10.1093 /$ nar/26.19.4413

6. Chaplin T, Bernard O, Beverloo HB, Saha V, Hagemeijer A, Berger R, et al. The T(10;11) Translocation in Acute Myeloid Leukemia (M5) Consistently Fuses the Leucine Zipper Motif of AF10 Onto the HRX Gene. Blood (1995) 86 (6):2073-6. doi: 10.1182/blood.V86.6.2073.bloodjournal8662073

7. Deshpande AJ, Deshpande A, Sinha AU, Chen L, Chang J, Cihan A, et al. AF10 Regulates Progressive H3K79 Methylation and HOX Gene Expression in Diverse AML Subtypes. Cancer Cell (2014) 26(6):896-908. doi: 10.1016/ j.ccell.2014.10.009

8. Okada Y, Jiang Q, Lemieux M, Jeannotte L, Su L, Zhang Y. Leukaemic Transformation by CALM-AF10 Involves Upregulation of Hoxa5 by Hdot1l. Nat Cell Biol (2006) 8(9):1017-24. doi: 10.1038/ncb1464

9. Lin YH, Kakadia PM, Chen Y, Li YQ, Deshpande AJ, Buske C, et al. Global Reduction of the Epigenetic H3K79 Methylation Mark and Increased Chromosomal Instability in CALM-AF10-Positive Leukemias. Blood (2009) 114(3):651-8. doi: 10.1182/blood-2009-03-209395

10. Nakamura F, Maki K, Arai Y, Nakamura Y, Mitani K. Monocytic Leukemia With CALM/AF10 Rearrangement Showing Mediastinal Emphysema. Am J Hematol (2003) 72(2):138-42. doi: 10.1002/ajh.10265

11. Huh JY, Chung S, Oh D, Kang MS, Eom HS, Cho EH, et al. Clathrin Assembly Lymphoid Myeloid Leukemia-AF10-Positive Acute Leukemias: A Report of 2 Cases With a Review of the Literature. Korean J Lab Med (2010) 30(2):117-21. doi: 10.3343/kjlm.2010.30.2.117

12. Asnafi V, Radford-Weiss I, Dastugue N, Bayle C, Leboeuf D, Charrin C, et al. CALM-AF10 is a Common Fusion Transcript in T-ALL and is Specific to the TCRgammadelta Lineage. Blood (2003) 102(3):1000-6. doi: 10.1182/blood2002-09-2913

13. Borel C, Dastugue N, Cances-Lauwers V, Mozziconacci MJ, Prebet T, Vey N, et al. PICALM-MLLT10 Acute Myeloid Leukemia: A French Cohort of 18 Patients. Leukemia Res (2012) 36(11):1365-9. doi: 10.1016/j.leukres.2012.07.008
(JH), the Children's Leukemia Research Association (JH), the Pediatric Cancer Research Fund, the Keegan Bradley Charity Golf Classic, the University of Vermont Health Network Group (JH), and the Charlotte Perelman Fund (GS). Donna and Martin Waldron were not involved in the study design, collection, analysis, interpretation of data, the writing of this article or the decision to submit it for publication.

\section{ACKNOWLEDGMENTS}

The flow cytometry was performed in the Harry Hood Bassett Flow Cytometry and Cell Sorting (FCCS) Facility at the University of Vermont Larner College of Medicine. We acknowledge Roxana del Rio-Guerra PhD, SCYM (ASCP) for her expertise and guidance during these experiments.

14. Williams MT, Yousafzai Y, Cox C, Blair A, Carmody R, Sai S, et al. Interleukin-15 Enhances Cellular Proliferation and Upregulates CNS Homing Molecules in Pre-B Acute Lymphoblastic Leukemia. Blood (2014) 123(20):3116-27. doi: 10.1182/blood-2013-05-499970

15. Yao H, Price TT, Cantelli G, Ngo B, Warner MJ, Olivere L, et al. Leukaemia Hijacks a Neural Mechanism to Invade the Central Nervous System. Nature (2018) 560(7716):55-60. doi: 10.1038/s41586-018-0342-5

16. Kupsa T, Horacek JM, Jebavy L. The Role of Cytokines in Acute Myeloid Leukemia: A Systematic Review. BioMed Pap Med Fac Univ Palacky Olomouc Czech Repub (2012) 156(4):291-301. doi: 10.5507/bp.2012.108

17. Becker PS. Dependence of Acute Myeloid Leukemia on Adhesion Within the Bone Marrow Microenvironment. TheScientificWorldJournal (2012) 2012:856467. doi: 10.1100/2012/856467

18. Jo DY, Rafii S, Hamada T, Moore MA. Chemotaxis of Primitive Hematopoietic Cells in Response to Stromal Cell-Derived Factor-1. J Clin Invest (2000) 105(1):101-11. doi: 10.1172/JCI7954

19. Busillo JM, Benovic JL. Regulation of CXCR4 Signaling. Biochim Biophys Acta (2007) 1768(4):952-63. doi: 10.1016/j.bbamem.2006.11.002

20. Konoplev S, Jorgensen JL, Thomas DA, Lin E, Burger J, Kantarjian HM, et al. Phosphorylated CXCR4 is Associated With Poor Survival in Adults With BAcute Lymphoblastic Leukemia. Cancer (2011) 117(20):4689-95. doi: $10.1002 / \mathrm{cncr} .26113$

21. Rombouts EJ, Pavic B, Lowenberg B, Ploemacher RE. Relation Between CXCR-4 Expression, Flt3 Mutations, and Unfavorable Prognosis of Adult Acute Myeloid Leukemia. Blood (2004) 104(2):550-7. doi: 10.1182/blood-2004-02-0566

22. Crazzolara R, Kreczy A, Mann G, Heitger A, Eibl G, Fink FM, et al. High Expression of the Chemokine Receptor CXCR4 Predicts Extramedullary Organ Infiltration in Childhood Acute Lymphoblastic Leukaemia. Br J Haematol (2001) 115(3):545-53. doi: 10.1046/j.1365-2141.2001.03164.x

23. Schneider CA, Rasband WS, Eliceiri KW. NIH Image to ImageJ: 25 Years of Image Analysis. Nat Methods (2012) 9(7):671-5. doi: 10.1038/nmeth.2089

24. Ortiz-Lazareno PC, Bravo-Cuellar A, Lerma-Diaz JM, Jave-Suarez LF, AguilarLemarroy A, Dominguez-Rodriguez JR, et al. Sensitization of U937 Leukemia Cells to Doxorubicin by the MG132 Proteasome Inhibitor Induces an Increase in Apoptosis by Suppressing NF-Kappa B and Mitochondrial Membrane Potential Loss. Cancer Cell Int (2014) 14(1):13. doi: 10.1186/1475-2867-14-13

25. Cheong JW, Kim Y, Eom JI, Jeung HK, Min YH. Enhanced Autophagy in Cytarabine Arabinoside-Resistant U937 Leukemia Cells and its Potential as a Target for Overcoming Resistance. Mol Med Rep (2016) 13(4):3433-40. doi: 10.3892/mmr.2016.4949

26. Bach C, Buhl S, Mueller D, Garcia-Cuellar MP, Maethner E, Slany RK. Leukemogenic Transformation by HOXA Cluster Genes. Blood (2010) 115 (14):2910-8. doi: 10.1182/blood-2009-04-216606

27. Dik WA, Brahim W, Braun C, Asnafi V, Dastugue N, Bernard OA, et al. CALM-AF10+ T-ALL Expression Profiles are Characterized by Overexpression of HOXA and BMI1 Oncogenes. Leukemia (2005) 19 (11):1948-57. doi: 10.1038/sj.leu.2403891 
28. Klein BJ, Deshpande A, Cox KL, Xuan F, Zandian M, Barbosa K, et al. The Role of the PZP Domain of AF10 in Acute Leukemia Driven by AF10 Translocations. Nat Commun (2021) 12(1):4130. doi: 10.1038/s41467-021-24418-9

29. Peled A, Petit I, Kollet O, Magid M, Ponomaryov T, Byk T, et al. Dependence of Human Stem Cell Engraftment and Repopulation of NOD/SCID Mice on CXCR4. Science (1999) 283(5403):845-8. doi: 10.1126/science.283.5403.845

30. Caudell D, Aplan PD. The Role of CALM-AF10 Gene Fusion in Acute Leukemia. Leukemia (2008) 22(4):678-85. doi: 10.1038/sj.leu.2405074

31. Sison EA, McIntyre E, Magoon D, Brown P. Dynamic ChemotherapyInduced Upregulation of CXCR4 Expression: A Mechanism of Therapeutic Resistance in Pediatric AML. Mol Cancer Res: MCR (2013) 11(9):1004-16. doi: 10.1158/1541-7786.MCR-13-0114

32. Zeng Z, Shi YX, Samudio IJ, Wang RY, Ling X, Frolova O, et al. Targeting the Leukemia Microenvironment by CXCR4 Inhibition Overcomes Resistance to Kinase Inhibitors and Chemotherapy in AML. Blood (2009) 113(24):6215-24. doi: 10.1182/blood-2008-05-158311

33. Wang S, Wang X, Liu S, Zhang S, Wei X, Song Y, et al. The CXCR4 Antagonist, AMD3100, Reverses Mesenchymal Stem Cell-Mediated Drug Resistance in Relapsed/Refractory Acute Lymphoblastic Leukemia. Oncol Targets Ther (2020) 13:6583-91. doi: 10.2147/OTT.S249425

34. Kremer KN, Peterson KL, Schneider PA, Meng XW, Dai H, Hess AD, et al. CXCR4 Chemokine Receptor Signaling Induces Apoptosis in Acute Myeloid Leukemia Cells via Regulation of the Bcl-2 Family Members Bcl-XL, Noxa, and Bak. J Biol Chem (2013) 288(32):22899-914. doi: 10.1074/jbc.M113.449926

35. Juarez J, Dela Pena A, Baraz R, Hewson J, Khoo M, Cisterne A, et al. CXCR4 Antagonists Mobilize Childhood Acute Lymphoblastic Leukemia Cells Into the Peripheral Blood and Inhibit Engraftment. Leukemia (2007) 21(6):124957. doi: 10.1038/sj.leu.2404684

36. Stuckel AJ, Zhang W, Zhang X, Zeng S, Dougherty U, Mustafi R, et al. Enhanced CXCR4 Expression Associates With Increased Gene Body 5Hydroxymethylcytosine Modification But Not Decreased Promoter Methylation in Colorectal Cancer. Cancers (Basel) (2020) 12(3):539-56. doi: $10.3390 /$ cancers 12030539

37. Jost TR, Borga C, Radaelli E, Romagnani A, Perruzza L, Omodho L, et al. Role of CXCR4-Mediated Bone Marrow Colonization in CNS Infiltration by T Cell Acute Lymphoblastic Leukemia. J Leukoc Biol (2016) 99(6):1077-87. doi: 10.1189/jlb.5MA0915-394R

38. Alsayed Y, Ngo H, Runnels J, Leleu X, Singha UK, Pitsillides CM, et al. Mechanisms of Regulation of CXCR4/SDF-1 (CXCL12)-Dependent Migration and Homing in Multiple Myeloma. Blood (2007) 109(7):2708-17. doi: 10.1182/blood-2006-07-035857
39. Sola A, Bertacchini J, D’Avella D, Anselmi L, Maraldi T, Marmiroli S, et al. Development of Solvent-Casting Particulate Leaching (SCPL) Polymer Scaffolds as Improved Three-Dimensional Supports to Mimic the Bone Marrow Niche. Mater Sci Eng C Mater Biol Appl (2019) 96:153-65. doi: 10.1016/j.msec.2018.10.086

40. Heath JL, Weiss JM, Lavau CP, Wechsler DS. Effects of Iron Depletion on CALM-AF10 Leukemias. Exp Hematol (2014) 42(12):1022-30 e1. doi: 10.1016/j.exphem.2014.08.004

41. Cooper TM, Sison EAR, Baker SD, Li L, Ahmed A, Trippett T, et al. A Phase 1 Study of the CXCR4 Antagonist Plerixafor in Combination With High-Dose Cytarabine and Etoposide in Children With Relapsed or Refractory Acute Leukemias or Myelodysplastic Syndrome: A Pediatric Oncology Experimental Therapeutics Investigators' Consortium Study (POE 10-03). Pediatr Blood Cancer (2017) 64(8):e26414-33. doi: 10.1002/pbc.26414

42. Martinez-Cuadron D, Boluda B, Martinez P, Bergua J, Rodriguez-Veiga R, Esteve J, et al. A Phase I-II Study of Plerixafor in Combination With Fludarabine, Idarubicin, Cytarabine, and G-CSF (PLERIFLAG Regimen) for the Treatment of Patients With the First Early-Relapsed or Refractory Acute Myeloid Leukemia. Ann Hematol (2018) 97(5):763-72. doi: 10.1007/s00277018-3229-5

43. Roboz GJ, Ritchie EK, Dault Y, Lam L, Marshall DC, Cruz NM, et al. Phase I Trial of Plerixafor Combined With Decitabine in Newly Diagnosed Older Patients With Acute Myeloid Leukemia. Haematologica (2018) 103(8):130816. doi: 10.3324/haematol.2017.183418

Conflict of Interest: The authors declare that the research was conducted in the absence of any commercial or financial relationships that could be construed as a potential conflict of interest.

Publisher's Note: All claims expressed in this article are solely those of the authors and do not necessarily represent those of their affiliated organizations, or those of the publisher, the editors and the reviewers. Any product that may be evaluated in this article, or claim that may be made by its manufacturer, is not guaranteed or endorsed by the publisher.

Copyright (c) 2022 Fertal, Zaidi, Stein, Stein and Heath. This is an open-access article distributed under the terms of the Creative Commons Attribution License (CC BY). The use, distribution or reproduction in other forums is permitted, provided the original author(s) and the copyright owner(s) are credited and that the original publication in this journal is cited, in accordance with accepted academic practice. No use, distribution or reproduction is permitted which does not comply with these terms. 